\title{
Práticas adotadas pela enfermagem para a promoção do cuidado desenvolvimental dos recém-nascidos prematuros
}

\author{
Practices adopted by nursing to promote developmental care for premature newborns \\ Prácticas adoptadas por la enfermería para promover el cuidado del desarrollo de los recién \\ nacidos prematuros
}

Recebido: 17/03/2021 | Revisado: 22/03/2021 | Aceito: 05/04/2021 | Publicado: 15/04/2021

Thiago Camilo Vaz de Sousa Lima
ORCID: https://orcid.org/0000-0002-3929-3344
Universidade de Brasília, Brasil
E-mail: thiagocamiloo@ hotmail.com
Rita de Cássia Melão de Morais
ORCID: https://orcid.org/0000-0001-8526-0642
Universidade de Brasília, Brasil
E-mail: ritamelao@unb.br
Aline Oliveira Silveira
ORCID: https://orcid.org/0000-0003-4470-7529
Universidade de Brasília, Brasil
E-mail: alinesilveira@ unb.br
Camila Camargo Medeiros
ORCID: https://orcid.org/0000-0002-4930-3817
Universidade de Brasília, Brasil
E-mail: camilacmedeiros@live.com
Elainne Motta
ORCID: https://orcid.org/0000-0003-3310-0094
Hospital Universitário de Brasília, Brasil
E-mail: elainne.motta@ebserh.gov.br

\begin{abstract}
Resumo
Objetivo: Descrever os conhecimentos e as práticas adotadas pela equipe de enfermagem para a promoção do cuidado desenvolvimental do recém-nascido pré-termo assistido na Unidade de Terapia Intensiva Neonatal. Metodologia: Estudo com abordagem qualitativa. O local do estudo foi a Unidade de Terapia Intensiva Neonatal de um Hospital público do Distrito Federal, em que participaram 12 profissionais de enfermagem, atuantes na unidade. Para a coleta de dados, foi utilizada entrevista semiestruturada e formulário de caracterização dos participantes. A análise dos dados foi temática. Resultados: Três unidades temáticas foram identificadas: Entendimento dos profissionais de enfermagem acerca do cuidado desenvolvimental; Cuidados de enfermagem na manipulação do recém-nascido pré-termo e; Influência do ambiente na evolução neurodesenvolvimental do recém-nascido pré-termo. Conclusão: Concluímos com esse estudo que a equipe de enfermagem da UTIN apresenta conhecimento sobre a promoção do desenvolvimento de recém-nascidos pré-termos dentro de uma unidade de terapia intensiva neonatal e conhecimento sobre as repercussões fisiológicas negativas, que podem afetar o RNPT, caso a assistência de enfermagem implementada não siga a filosofia do cuidado desenvolvimental.
\end{abstract}

Palavras-chave: Terapia intensiva neonatal; Enfermagem neonatal; Prematuro; Crescimento e desenvolvimento; Cuidados de enfermagem.

\begin{abstract}
Objective: To describe the knowledge and practices adopted by the nursing team to promote the developmental care of preterm newborns assisted in the Neonatal Intensive Care Unit. Methodology: Study with a qualitative approach. The study site took place at the Neonatal Intensive Care Unit of a public hospital in the Federal District-Brazil, in which participated 12 nursing professionals who work at the unit. For data collection, the semi-structured interview and the participants' characterization form were used. Data analysis was thematic. Results: Three thematic units were identified: Understanding of nursing professionals about developmental care, nursing care in handling the preterm newborn and influence of the environment on the neurodevelopmental evolution of the preterm newborn. Conclusion: We concluded with this study that the NICU nursing team has knowledge about promoting the development of preterm newborns within a neonatal intensive care unit. They are also aware of the negative physiological repercussions that can affect PTNB, in case the implemented nursing care does not follow the philosophy of developmental care.
\end{abstract}


Keywords: Intensive care; Neonatal; Neonatal nursing, Infant; Premature; Growth and development; Nursing care.

\section{Resumen}

Objetivo: Describir los conocimientos y prácticas adoptadas por el equipo de enfermería para promover el cuidado del desarrollo de los recién nacidos prematuros atendidos en la Unidad de Cuidados Intensivos Neonatales. Metodología: Estudio con enfoque cualitativo. El lugar de estudio fue la Unidad de Cuidados Intensivos Neonatales de un hospital público del Distrito Federal-Brazil, participaron del estúdio 12 profesionales de enfermería que laboran en la unidad. Para la recolección de datos se utilizó la entrevista semiestructurada y el formulario de caracterización de los participantes. El análisis de datos fue temático. Resultados: Se identificaron tres unidades temáticas: Comprensión de los profesionales de enfermería sobre los cuidados del desarrollo, cuidados de enfermería en el manejo del recién nacido prematuro e influencia del entorno en la evolución del neurodesarrollo del recién nacido prematuro. Conclusión: Con este estudio concluimos que el equipo de enfermería de la UCIN tiene conocimientos sobre la promoción del desarrollo del recién nacido prematuro dentro de una unidad de cuidados intensivos neonatales y conocimiento sobre las repercusiones fisiológicas negativas que pueden afectar a la PTNB, si el cuidado de enfermería implementado no sigue la filosofía del cuidado del desarrollo.

Palabras clave: Cuidado intensivo neonatal; Enfermería neonatal prematuro; Crecimiento y desarrollo; Atención de enfermería.

\section{Introdução}

O termo prematuro é utilizado para definir os recém-nascidos (RN), nascidos antes de completar 37 semanas de idade gestacional. Em 2018, cerca de 10,9\% dos 2.944 .932 nascimentos registrados no Brasil foram de prematuros (Ministério da Saúde, 2020). Tais RN prematuros são usualmente encaminhados à Unidade de Terapia Intensiva Neonatal (UTIN) para serem assistidos durante o período de transição precoce ao ambiente extrauterino, por necessitarem de se manter estáveis, num ambiente rico em estímulos. Sendo assim, a promoção de um ambiente mais estável a esses bebês é essencial para a facilitação do desenvolvimento normal da criança. Os cuidados no desenvolvimento da criança durante o período neonatal influenciam positivamente no desenvolvimento neuromotor (Barradas et al., 2006).

$\mathrm{O}$ avanço do conhecimento e do desenvolvimento tecnológico na área da terapia intensiva neonatal, aliado à sofisticação dos recursos terapêuticos, permitiu o aumento da sobrevida do RN, especialmente de prematuros (Kegler et al., 2016), porém, fatores como o aumento de equipamentos mais modernos e do número de procedimentos invasivos com a necessidade constante de luz, ruído ambiente e a manipulação excessiva durante o cuidado, ocasionam uma série de efeitos adversos que desencadeiam alterações no desenvolvimento dos neonatos, principalmente dos recém-nascidos pré-termo (RNPT) (Costa \& Calado, 2019).

Os RN prematuros são considerados de risco biológico para atraso global do desenvolvimento. O desenvolvimento infantil é baseado em domínios de funções relacionadas aos aspectos motores, cognitivos e linguísticos, influenciados por fatores biológicos, psicossociais e ambientais. Crianças que nascem prematuras podem apresentar um atraso em diferentes domínios do desenvolvimento, por exemplo, o motor, o adaptativo, a cognição e a linguagem, mesmo que os déficits nestas áreas sejam mais sutis (Ribeiro et al., 2017).

Na década de 1980, Heidelise Als desenvolveu uma modalidade de proteção ao desenvolvimento dos RNPT, denominada Neonatal Individualized Developmental Care and Assessment Program (NIDCAP), que consiste em melhorar o ambiente das unidades de terapia intensiva, como a redução da luminosidade, ruído e manuseio, combinados com a promoção de períodos de descanso para o RN, com a finalidade de diminuir o alto nível de estresse ambiental (Moody et al., 2017).

Os RNPT submetidos ao NIDCAP no período neonatal apresentaram melhora significativa na regulação dos sistemas motor e de autorregulação avaliados pelo Assessment of Preterm Infants' Behavior (APIB), além de melhor desenvolvimento neurológico avaliado pelo Prechtl Neurologic Examination of the Fullterm Newborn Infant, em comparação aos que receberam o cuidado de rotina da UTIN, com duas semanas de idade corrigida (Moody et al., 2017).

O enfermeiro tem um papel primordial no cuidado de RNPT em uma UTIN, pois está em contato integral com os 
Research, Society and Development, v. 10, n. 4, e39010413993, 2021

(CC BY 4.0) | ISSN 2525-3409 | DOI: http://dx.doi.org/10.33448/rsd-v10i4.13993

bebês, do nascimento ao momento da alta. Um RNPT necessita de atenção e cuidados especiais, o que faz de uma UTIN um local que exige a atuação de profissionais responsáveis com o cuidar, comprometidos e capacitados, conciliando a competência, agilidade, destreza técnica e experiência clínica, com a sensibilidade para perceber as necessidades individuais de cada neonato (Câmara, Pereira \& Silva, 2019). A atuação em UTI neonatal e pediátrica exige a aplicação de uma assistência permeada por aparatos tecnológicos e monitorização constante sob um nível de estresse tanto para os profissionais quanto para as crianças e familiares (Reis et al., 2013).

A literatura evidencia que o enfermeiro está diretamente ligado à assistência e é elemento obrigatório, no quadro assistencial da UTIN, tendo um papel fundamental na oferta de um cuidado qualificado, humanizado e integral ao recémnascido crítico, devendo assim considerar o cuidado desenvolvimental na sua prática assistencial (Reis et al., 2013). Os estudos apontam que as intervenções em cuidado ao desenvolvimento promoveram melhora a curto prazo nos resultados fisiológicos, comportamentais, neurológicos e clínicos dos recém-nascidos (Moody et al., 2017).

O presente estudo teve como base conceitual o "Cuidado Desenvolvimental", filosofia de cuidado que objetiva minimizar o estresse gerado pelo ambiente de terapia intensiva e prevenir complicações, a curto e a longo prazo, ao RN crítico e a sua família. Nesse sentido, esse conceito tem como princípio uma assistência estruturada que suporta, orienta e incentiva a organização e desenvolvimento do cuidado, a fim de identificar e agir sobre suas fragilidades físicas, emocionais e psicológicas. Somado ao fato de reduzir os impactos deletérios no desenvolvimento neurológico, durante a internação e ao uso de ventilação mecânica, como também favorecer o ganho de peso e aumentar a participação dos pais nos cuidados de seu filho(a) (Lisanti, Connock \&Medoff-Cooper, 2016). Os princípios básicos do cuidado desenvolvimental são: posicionamento, estimulo do contato pele-a-pele desde que com segurança, cuidado agrupado, tratamento não farmacológico da dor, gestão ambiental e alimentação (Lisanti et al, 2016; Marski et al., 2018).

Diante do exposto e da importância do profissional de enfermagem nos cuidados do RNPT na UTIN, elaboramos a seguinte questão norteadora: Quais as práticas adotadas pelos profissionais de enfermagem para promover o cuidado desenvolvimental do RNPT assistido na UTIN? Como objeto do estudo a "práticas do enfermeiro para promoção do cuidado desenvolvimental ao RNPT". Neste sentido, o objetivo da pesquisa foi descrever os conhecimentos e práticas adotadas pela equipe de enfermagem para a promoção do cuidado desenvolvimental do recém-nascido pré-termo assistido na Unidade de Terapia Intensiva Neonatal.

\section{Metodologia}

Trata-se de um estudo exploratório, de abordagem qualitativa, realizado na Unidade de Terapia Intensiva Neonatal do Setor Materno-infantil de um Hospital Público do Distrito Federal. Segundo Minayo (2014) a pesquisa qualitativa responde a questões particulares e aborda um nível de realidade que não pode ou não deveria ser quantificado. Neste sentido, trata-se de um universo de significados, motivos, aspirações, crenças, valores e atitudes.

Participaram do estudo enfermeiros e técnicos de enfermagem atuantes na Unidade de Terapia Intensiva Neonatal. Os critérios de inclusão foram enfermeiros e técnicos de enfermagem, lotados na UTIN. Como critérios de exclusão, profissionais que estavam de férias ou licença ou ainda, que pertenciam a outros setores se encontram temporariamente na unidade. Os participantes foram identificados como TE (técnicas de enfermagem) e Enf (enfermeiras), seguido dos números cardinais representando a ordem das entrevistas.

Para a obtenção dos dados, foram utilizados dois instrumentos: o formulário de caracterização dos profissionais de enfermagem da UTIN e entrevista semiestruturada. As entrevistas foram realizadas no próprio setor em horários que não atrapalharam a rotina do serviço e do profissional. Foi utilizado um instrumento previamente elaborado com perguntas abertas 
que abordaram aspectos referentes ao entendimento dos profissionais de enfermagem sobre o cuidado desenvolvimental do RNPT; as condutas adotadas pelos profissionais de enfermagem para minimizar a influência do ambiente no desenvolvimento do RNPT na UTIN e as condutas para promoção desenvolvimental do RNPT.

Para a captação dos participantes, o pesquisador deslocou-se à UTIN e consultou os profissionais de enfermagem sobre o interesse em participar do estudo. Quando os participantes reportaram interesse e aceitação, o pesquisador agendava o melhor momento para realização da pesquisa na conveniência dos mesmos, não comprometendo o seu processo de trabalho. $\mathrm{O}$ local da coleta de dados foi no repouso da enfermagem, proporcionando privacidade e ausência de movimentação de transeuntes.

A coleta dos dados ocorreu de agosto a outubro de 2019, sendo finalizada quando os pesquisadores atingiram a saturação teórica dos dados, ou seja, quando nenhum novo elemento foi encontrado e o acréscimo de novas informações deixou de ser necessário, pois não alterava a compreensão do fenômeno estudado (Nascimento et al., 2018).

No sentido de validar o roteiro de entrevista semiestruturada (validação interna) o pesquisador realizou, previamente, duas entrevistas, uma buscando atender aos objetivos do estudo e outra no ajuste as perguntas a fim de evitar interpretações ambíguas, o que poderia comprometer o rigor do método, na obtenção dos dados. Após a validação do instrumento iniciou-se a coleta dos resultados.

As entrevistas foram gravadas com intuito de não ocorrer uma perda de resultados significativos e posteriormente transcritas pelo próprio pesquisador. A análise de dados foi realizada pela análise de conteúdo da modalidade "temática" de acordo com os preceitos de Minayo (2014), seguindo as três etapas recomendadas, quais sejam: pré-análise, exploração do material e tratamento dos resultados e interpretação. Desvelando três unidades temáticas intituladas: Entendimento dos profissionais de enfermagem acerca cuidado desenvolvimental, cuidados de enfermagem na manipulação do recém-nascido pré-termo e influência do ambiente na evolução neurodesenvolvimental do recém-nascido pré-termo;

Em relação aos procedimentos éticos em pesquisas com seres humanos, o projeto de pesquisa foi aprovado pelo Comitê de Ética em Pesquisa em Seres Humanos da Faculdade de Ciências da Saúde da Universidade de Brasília, com o parecer de número: 3.414.119, seguindo as recomendações da Resolução 466/2012 do Conselho Nacional de Saúde. Todos os participantes que aceitaram participar do estudo, assinaram o Termo de Consentimento Livre e Esclarecido (Ministério da Saúde, 2012).

Para manter o rigor metodológico do estudo, foi utilizada a lista de critérios consolidados para pesquisa qualitativa (COREQ) como ferramenta de apoio. Constitui-se de 32 itens de verificação relacionados à: equipe de pesquisa; projeto de pesquisa; e análise dos dados em relação a métodos de pesquisa qualitativa (Tong, Sainsbury \& Craig, 2015).

\section{Resultados e Discussão}

\subsection{Caracterização dos profissionais de enfermagem da UTIN}

Foram entrevistadas 12 profissionais de Enfermagem da Unidade de Terapia Intensiva Neonatal do Hospital Universitário, todas do sexo feminino, sendo três enfermeiras e nove técnicas de enfermagem. A média de idade entre as participantes foi de 39 anos, variando de 28 a 52 anos. A média de tempo de experiência profissional foi de 10 anos, especificamente em neonatologia foi de cinco anos e cinco meses e na UTIN dessa instituição foi de três anos e um mês. Dessas entrevistadas, dez participantes relataram que esse tema foi abordado esse tema durante sua formação e dois disseram que foi parcialmente abordado. Os três principais meios utilizados, pelas entrevistadas para se atualizarem sobre o cuidado desenvolvimental do RNPT, foram a internet, artigos científicos e cursos. Das 12 entrevistadas, oito relataram que já participaram de algum curso ou treinamento promovido pela instituição, e somente uma relatou não haver protocolos ou 
Research, Society and Development, v. 10, n. 4, e39010413993, 2021

(CC BY 4.0) | ISSN 2525-3409 | DOI: http://dx.doi.org/10.33448/rsd-v10i4.13993

diretrizes sobre o tema na unidade da instituição.

Constata-se que o perfil dos profissionais de enfermagem deste estudo é predominante feminino, sendo a maioria da categoria técnicos de enfermagem, que atuam na UTIN há bastante tempo, consequentemente, com uma abundante experiência na área de neonatologia, acrescem-se ainda, o fato de que tiveram o conteúdo referente ao cuidado desenvolvimental do RNPT na sua formação profissional e/ou no seu local de trabalho. Os dados identificados corroboram com o estudo de Anversa et al., (2011) a predominância de mulheres na UTIN, com percentual acima de 90\%. A categoria predominante é de técnicos de enfermagem (55,5\%), os profissionais buscaram aperfeiçoamento profissional, especializando-se na área. Quanto ao tempo de atuação na área de neonatologia, um percentual acima de 35\% possui, em média, 20-25 anos, seguido de 45\% entre 10-20 anos com os demais estando há menos de 10 anos na referida unidade.

\subsection{Unidades temáticas}

\section{Unidade 1: Entendimento dos profissionais de enfermagem acerca Cuidado Desenvolvimental}

Foi abordado o entendimento dos profissionais de enfermagem quanto ao cuidado desenvolvimental ofertado ao RNPT, durante sua internação na UTIN, assim como quais os benefícios para o bom desenvolvimento da bebê, como se pode verificar nas falas abaixo:

Eu entendo que isso inclui cuidados e práticas que favoreçam o desenvolvimento neurológico e o desenvolvimento do bebê como um todo, tipo posicionamento do bebê, controle e alívio da dor, a amenização de ruídos, amenização de estimulações excessivas, tanto de ruídos e o visual. (Enf1)

É um cuidado especializado pro RN. Visando os protocolos de neuroproteção. Respeitando o ciclo sono vigília do RN. Manuseio Mínimo. Seguindo o protocolo de cuidados agrupados. (Enf 2)

Acho que é toda a assistência que a gente dá pro RN visando o bom desenvolvimento dele dentro da unidade pra que ele tenha melhor desenvolvimento pra quando ele tiver alta. (TE3)

A equipe de enfermagem relata ter conhecimentos sobre o cuidado desenvolvimental para a promoção do bom desenvolvimento do RNPT. Há estudos em que os resultados revelaram que os enfermeiros detêm conhecimentos sobre grande parte dos aspectos implicados no Cuidado Desenvolvimental, reconhecem o mesmo como relevante a uma prática de qualidade e segura ao RN (Marski et al., 2018). Há uma correlação à qualidade de vida e saúde das crianças após tratamento em unidades de terapia intensiva, com a assistência recebida dentro da UTIN. Esta é de extrema importância para uma vida pós-alta com menos sequelas e uma vida igual àqueles que não passaram pela prematuridade (Mestrovic et al., 2008).

Alguns profissionais ressaltam que o ambiente da UTIN deve ser equivalente ao ambiente intraútero para proporcionar ao RNPT a oportunidade de completar sua maturação extraútero, como nas falas descritas:

Bom, o RN ele na verdade deveria estar no útero ainda, no maior conforto possível, então a gente precisa manter o repouso, o silencio, o manuseio, dependendo da prematuridade o manuseio tem que ser mínimo, tem que ter cuidado com luxação de quadril, com pressão intracraniana, ter um mínimo de manuseio e promover o máximo de conforto possivel pra esse RN. (TE7)

Cuidado é ele desenvolver o que ele não desenvolveu o que era pra ser desenvolvido dentro da barriga, desenvolver 
Research, Society and Development, v. 10, n. 4, e39010413993, 2021

(CC BY 4.0) | ISSN 2525-3409 | DOI: http://dx.doi.org/10.33448/rsd-v10i4.13993

fora mas com todo nosso apoio e cuidado dentro da UTI." (TE5)

Alguns RNPT precisam de assistência especializada em razão de suas condições clínicas. Assim sendo, necessitam de um ambiente apropriado, com recursos tecnológicos e humanos adequados, para garantir o tratamento e restabelecimento, pois o RN prematuro pode necessitar de hospitalização para que se adapte ao ambiente extrauterino. A equipe de enfermagem é responsável pela promoção e adaptação do RN ao meio externo com a oferta de um ambiente com equilíbrio térmico adequado, umidade, luz, som e estímulo cutâneo; observando o quadro clínico; monitorando os sinais e o desenvolvimento da terapêutica adotada ao RN; tentar atender às necessidades do mesmo, da mãe e supervisionar os cuidados de enfermagem prestados (Ribeiro et al., 2016).

\section{Unidade 2: Cuidados de enfermagem na manipulação do RNPT}

Esta unidade temática tratará das seguintes subunidades temáticas: Minimizar manipulação e concentrar o cuidado, prevenção de hemorragia intracraniana, Posicionamento do RNPT no leito e a Troca de Fraldas.

\section{Minimizar manipulação e concentrar o cuidado}

A equipe de enfermagem valoriza a manipulação mínima dos RNPT para promoção do desenvolvimento neurológico, assim como, o cuidado prestado entre a equipe multiprofissional seja agrupado, com o objetivo de manipular o mínimo possível o bebê, como relatado pelos profissionais:

Tentar padronizar o máximo a questão de manipulação do RN, a equipe é multiprofissional, tem várias especialidades. Tentar adequar a esse serviço, porque tipo assim, a fisioterapeuta [...] o médico [...] a enfermagem vem e mexe, dai a pouco coleta exame, então assim, tentar minimizar essa manipulação. (TE1)

[...] muitos RNPT menores que 34 semanas, eles têm uma vulnerabilidade muito grande sensorial, então a gente ao tocar o bebê, a forma que a gente toca, e a forma que a gente lava e faz a higienização deve ser regrado pra que a gente não cause ali um trauma ou estímulo sensorial desnecessário para formação do nenê [...] E por último na manipulação, então a forma como você manipula, como você muda o decúbito, como você toca no bebê. (TE9)

Tem uns bebês que são manuseio mínimo 1, outros manuseio mínimo 2 e outros manuseio mínimo normal [...] $O$ cuidado em manusear o RN... Tudo isso pra evitar um dano irreversível no cérebro do bebê, principalmente na questão do manuseio do bebê [...] Manuseio mínimo com os RN, os cuidados com eles [...] (TE8)

Nas primeiras 72 horas manter o bebê na posição neutra. Protocolo de manuseio mínimo, cuidados agrupados com a equipe multidisciplinar pra não ficar muitas pessoas toda hora entrando. (Enf3)

Nos relatos prestados, os profissionais de enfermagem demonstram preocupação com a manipulação excessiva do RNPT, bem como, a necessidade da organização da assistência pela equipe multiprofissional de forma que esta seja agrupada para manipular o mínimo ao RN e prevenir exposições que possam ser prejudiciais ao desenvolvimento do RNPT.

Esses achados corroboram com a literatura que para melhorar a qualidade do atendimento ao RN, faz-se premente o cuidado desenvolvimental como uma filosofia de assistência que implica repensar as relações com o RN, a família e os sujeitos envolvidos no cuidado. Entre as várias estratégias do cuidado desenvolvimental, o agrupamento de cuidados tem como 
objetivo promover o repouso do RN por meio da manipulação mínima e deve ser realizado com o intuito de diminuir o gasto energético e o estresse (Lisanti el al., 2016; Marques et al., 2017).

O sono para os RNPT constitui uma importante necessidade básica, como a maturação do sistema nervoso central, consolidação da memória, aprendizado, preservação da energia, termorregulação, imunidade, acrescentando-se ainda, o estímulo à produção proteica e de alguns hormônios. Logo a manipulação excessiva priva o sono dos RNPT podendo prejudicar o seu desenvolvimento neuropsicomotor (Maki el al., 2017).

As instituições de saúde têm o dever de evitar a dor e o estresse ao RNPT formulando previamente e implementando ações que favoreçam o manuseio mínimo como o agrupamento de cuidados, horários pré-estabelecidos para que não haja muita interrupção durante a estadia na UTIN e foco nos procedimentos realmente necessários para cada paciente, assim individualizando assim os cuidados (Magalhães et al., 2011).

\section{Prevenção de hemorragia intracraniana}

Outro aspecto abordado pelos profissionais de enfermagem foi com relação ao aos cuidados desenvolvimentais para preservar a pressão intracraniana com a finalidade de prevenir hemorragia intracraniana e possíveis sequelas.

[...] existe também uma maior predisposição do RNPT de ter hemorragia intraventricular e para que isso não aconteça algumas medidas de enfermagem são extremamente necessárias no manejo do RN pra poder evitar esse tipo de intercorrência indesejável. (TE9)

[...] pressão intracraniana, ter um mínimo de manuseio e promover o máximo de conforto possível pra esse RN [...] na troca de fraldas [...] o bebê lateralizado [...] eleva pressão intracraniana, porque quando você eleva o quadril o sanguinho vai todo pra cabeça e pode ter lesão e essas lesões variam de acordo com cada nível, nível menores acontece de absorver e outros não, e essa lesão grande pode gerar problemas pra esses RN pra vida inteira, então ter muito cuidado com o manuseio. (TE7)

Os profissionais de enfermagem deste estudo conhecem os cuidados específicos para a prevenção do aumento da pressão intracraniana e reconhecem as repercussões no caso de hemorragia intracraniana e quais consequências pode ter na vida do RNPT. O simples ato da troca de fraldas pode causar lesões.

Como o desenvolvimento neurológico do recém-nascido acontece de forma progressiva, prematuros estão mais sujeitos a lesões do sistema nervoso central, principalmente hemorragias peri e interventriculares que podem ocorrer devido a alterações na pressão arterial sistêmica determinadas por vezes, por posturas inadequadas (Monteiro et al., 2019; Santos et al., 2018).

\section{Posicionamento do RNPT no leito}

Para a promoção do cuidado desenvolvimental do RNPT, deve-se ter cuidado com o seu posicionamento adequado do RNPT, como ressalta as Enf1 e Enf3:

Eu entendo que isso inclui cuidados e práticas que favoreçam o desenvolvimento neurológico e o desenvolvimento do bebê como um todo, tipo posicionamento do bebê... nesse protocolo estão incluídos todos os cuidados que vão favorecer esse desenvolvimento, posicionamento adequado do bebê... no caso do prematuro extremo não alternar decúbito. (Enf1) 
Nas primeiras $72 \mathrm{hrs} \mathrm{manter} \mathrm{o} \mathrm{bebê} \mathrm{na} \mathrm{posição} \mathrm{neutra.} \mathrm{(Enf} \mathrm{3)}$

Verifica-se no relato da enfermeira que a instituição possuí protocolos específicos para a promoção desenvolvimental do RNPT, com um dos aspectos a serem abordados, a importância do posicionamento adequado do RN.

$\mathrm{O}$ posicionamento adequado do RN favorece a estabilidade do bebê, reduz o gasto energético, alinhamento musculoesquelético correto, promove a organização dos estágios de sono e vigília com consequente aumento do sono e fornece estímulos proprioceptivos adequados ao desenvolvimento do sistema nervoso central, e, portanto, são protetores ao desenvolvimento. Comportamentos que refletem uma estabilidade do subsistema motor incluem postura harmoniosa, com equilíbrio entre flexão e extensão, ausência de hipotonia ou hipertonia e movimentos sincrônicos e harmoniosos dos membros (Monteiro et al., 2019).

O posicionamento do RNPT no leito também interfere diretamente sobre o seu desenvolvimento neurocomportamental e motor, com respostas diretas sobre padrões comportamentais de organização, posturas em linha média e movimentos finos. Quanto à utilização de artefatos para a manutenção de posturas, foi salientado que o ninho não apenas promove a adoção de posturas flexoras, como também facilita a realização de movimentos finos, em direção a linha média, elementos que contribuem para o desenvolvimento neurocomportamental do RNPT (Santos et al., 2018; Lisanti et al, 2016).

\section{Unidade 3: A influência do ambiente na evolução neurodesenvolvimental do RNPT}

Outro aspecto importante que interfere no neurodesenvolvimento do RNPT são as questões ambientais da UNTIN, tais como: ruídos, incubadora, luminosidade e termorregulação. A UTIN é um ambiente que necessita de atenção especial e o ruído deste local pode afetar o desenvolvimento do RNPT, como verificado nos relatos abaixo:

A questão do barulho também, o nenê precisa pra se desenvolver correto ele precisa estar $90 \%$ do tempo dele em estado de sono e vigília, porque senão o desenvolvimento é retardado então a gente tenta manter o ambiente silencioso [...] (TE 9)

Os ruídos externos, que as vezes a gente meio que esquece que está numa uti neonatal e aumenta muito o tom de voz. Barulhos, até o pisado deve ser diferente, ne? (TE1)

Os profissionais de enfermagem relatam a importância de um ambiente silencioso para o bom desenvolvimento do RNPT, no entanto em algumas situações esse silencio não é preservado.

Foi constatado num estudo realizado no Brasil, foi constatado que os ruídos ocasionados pela manipulação dos RN repercutiram em modificações comportamentais e estresse (Costa \& Calado, 2020). O aumento da pressão sonora que é exposta aos RN é proporcional ao aumento dos efeitos negativos para os mesmos, levando ao aumento da pressão intracraniana, advinda da irritabilidade e do choro, aumentando a ocorrência de hemorragia intracraniana. Ainda como efeito indesejado temos o consumo maior de energia e a desaceleração do ganho de peso consequente do possível aumento do consumo de $\mathrm{O}_{2}$ e da elevação da frequência cardíaca (Rodarte et al., 2019).

É de tamanha importância manter a unidade de terapia intensiva neonatal um ambiente tranquilo e silencioso, o ruído é extremamente prejudicial para o desenvolvimento do RN no seu período de internação, deve-se estar particularmente atento ao tom da voz e manipulação de objetos que emitem muitos ruídos, como no controle do ambiente, devendo este estar interditado a circulação de pessoas às quais não sejam necessárias, pois por mais que se tente manter o silencio, estas acabam emergindo sons decorrentes de uma grande aglomeração, também é necessário informar aos pais e outros visitantes sobre a 
questão do excesso de ruídos.

Ainda com relação à influência do ambiente da UTIN no desenvolvimento do RNPT, outro fator citado pelos profissionais de enfermagem foi a luminosidade:

[...] a luminosidade tem que ser baixa [...] pelo menos tentar diminuir a luz. (TE5)

[...] botar sempre o blackout pra fazer com que essa criança tenha o ambiente o mais tranquilo possível pro desenvolvimento...menos estímulo visual, tipo de luz para que a criança possa se desenvolver corretamente. (TE9)

O efeito da luminosidade é bastante semelhante ao do ruído, pois atrapalha o sono e vigília do RN causando um ambiente estressante e hiper estimulante, que acarreta num atraso no desenvolvimento podendo causar danos irreversíveis.

Os achados são semelhantes aos identificados em estudos pertinentes em que os profissionais de algumas UTIN's usavam um cobertor em cima da incubadora para abafar o som e proteger da luminosidade (Costa \& Calado, 2020). Os autores afirmam ainda que a introdução de um período de repouso com luzes e ruídos amenizados, representando a "noite", possibilita a regulação dos sistemas corporais do neonato, diminuindo o gasto de energia e acelerando o ganho de peso (Lisanti et al., 2016).

Um ambiente agitado, ruidoso, com luz contínua e intensa, além de causar estresse ao bebê, pode alterar seus padrões fisiológicos e pode comprometer sua acuidade visual e auditiva, e embora cientificamente não haja associação entre luzes fortes contínuas e a retinopatia da prematuridade, a alta luminosidade compromete o sono e descanso do prematuro, impedindo-o de repousar ou dormir, causando irritabilidade, alterações na frequência cardíaca e respiratória, além de evitar que este abra os olhos em seus momentos de vigília (Costa \& Calado, 2020).

Algo que é afetado diretamente caso não haja um ambiente ideal para o desenvolvimento do RN, é a termorregulação, que nada mais é que a manutenção da temperatura corporal do neonato, como levantado por essa técnica de enfermagem:

[...] manter sempre a incubadora aquecida [...] a manipulação como é mínima tem que toda equipe, preferencialmente, entrar todo mundo junto pra manipular no mesmo momento pra evitar perda de calor, que aí também perde peso. (TE2)

\section{Incubadora aquecida e umidificada. (Enf3)}

A termorregulação consiste na manutenção ideal da temperatura corporal através de controle fisiológico. Os valores de normalidade para os recém-nascidos (RN) estão entre $36,5^{\circ}$ e $37,5^{\circ} \mathrm{C}$. Fora dessa faixa, pode se considerar uma ameaça à homeostase, podendo acarretando distúrbios metabólicos e outras intercorrências, aumentando o risco de morbimortalidade neonatal (Martins et al., 2019).

Os RN têm aptidão de controlar sua temperatura corpórea em situações normais de temperatura externa, porem quando colocados em situações em que a temperatura é muito extrema, tanto baixa quanto alta, há uma incapacidade de termorregulação por não manterem a homeostase. A exposição a hipotermia aguda causa vaso constrição periférica, podendo acarretar metabolismo anaeróbio, acidose adicionais e até mesmo vaso constrição pulmonar. Essas constatações reforçam a importância de se manter a incubadora sempre aquecida e tentar ao máximo agrupar os cuidados para evitar a abertura desnecessária da incubadora ou da perda de calor devido a gastos de energia proporcionados por manejos excessivos (Rolim et al., 2010).

Outro aspecto abordado pelos profissionais, que interfere no desenvolvimento do RNPT, foi sobre os ruídos da 
incubadora, como é expresso nas seguintes falas:

[...] a incubadoras também, vibração muito fortes na incubadora, a gente tem o péssimo hábito de ficar escrevendo na incubadora. (TE1)

[...] em questão das incubadoras, de não bater muito forte as portas essas questões assim podem interferir também. (TE2)

A questão da incubadora está ligada diretamente ao ambiente estressante, abordado nas subcategorias Ruído e Luminosidade e na importância da manutenção da temperatura abordada no tópico anterior. A incubadora é o templo do RN, que deve ser o mais semelhante possível ao útero da mãe, sendo um ambiente umidificado, aquecido, tranquilo, confortável, uma barreira física importante para o progresso do $\mathrm{RN}$ durante toda a sua internação.

Os ruídos a que os RN estão expostos dentro das incubadoras, durante as práticas do cuidado, podem acarretar estresse, modificar o estado comportamental e atrapalhar no estado de sono e vigília (Rodarte et al., 2019). Ainda, o ruído em níveis elevados pode acarretar danos sérios ao desenvolvimento do RNPT, em que quando presente, verifica-se os sinais de estresse, de apneia, respiração irregular, diminuição da oxigenação, aumento da frequência cardíaca e respiratória, palidez, náusea, vômitos, cianose, eructação, flacidez, tremores, irritabilidade e choro (Nazario et al, 2105).

Atualmente com o avanço das tecnologias hospitalares torna-se mais complicado em reduzir os ruídos existentes no ambiente, pois quanto mais equipamentos modernos no setor, mais efeitos sonoros são emitidos, como os alarmes (Santana $e t$ al., 2015). Segundo estudo, os níveis de ruídos dentro de uma UTIN são mais elevados do que é recomendado pela Academia Americana de Pediatria, que seria de 65dBA, e uma forma de diminuir essa exposição seria a adesão integral por parte dos profissionais aos protocolos, uma manipulação cuidadosa da incubadora, investigação de protocolos de estímulos sonoros adequados ou talvez a implementação de protetores auriculares de silicone para os RN (Rodarte et al., 2019).

\section{Limitações do Estudo}

Durante a pesquisa deparou-se com dificuldades na realização das entrevistas, como: resistência dos profissionais a responder o questionário, aparentemente devido à impressão de estarem sendo avaliados, além da falta de disponibilidade desses profissionais em participar do processo em alguns momentos, devido à grande demanda de serviço do setor.

\section{Considerações Finais}

Concluímos com o estudo que a equipe de enfermagem da UTIN apresenta conhecimento sobre a promoção do desenvolvimento de recém-nascidos pré-termos dentro de uma unidade de terapia intensiva neonatal. Assim como, ela tem conhecimento sobre as repercussões fisiológicas negativas que podem afetar o RNPT, caso a assistência de enfermagem implementada não siga a filosofia do cuidado desenvolvimental. Esta investigação traz o significado atribuído pelos profissionais de enfermagem diante da realidade estudada, sobre o cuidado desenvolvimental que interferem diretamente na prática assistencial de enfermagem aos recém-nascidos prematuros hospitalizados na UTIN. Contudo, verificou-se que alguns profissionais de enfermagem abordam o tema, sem embasamento científico, com base apenas nos conhecimentos adquiridos durante a sua atuação profissional. 


\section{Referências}

Anversa, R. S. M., Ubessi, L. D., \& Stumm, E. M. F. (2011). Perfil de profissionais de terapia intensiva neonatal relacionado com estresse. Revista Baiana de Enfermagem, 25(3), 269-276.

Barradas, J. et al. (2006). A relação entre posicionamento do prematuro no Método Mãe-Canguru e desenvolvimento neuropsicomotor precoce. J. Pediatr, $82(6), 475-480$.

Câmara, T., Pereira, N., \& Silva, R. (2019). Enfermagem e o manuseio do recém-nascido na unidade de terapia intensiva neonatal. Revista Uningá, 56(S2), $222-233$.

Costa, M., \& Calado, G. (2020). O ambiente terapêutico e o desenvolvimento do recém-nascido prematuro: uma revisão integrativa da literatura. Revista Ibero-Americana de Saúde e Envelhecimento, 5(3), 1934.

Kegler, J. J., Paula, C. C., Neves, E. T., \& Jantsch, L. B. (2016). Manejo da dor na utilização do cateter central de inserção periférica em neonatos. Escola Anna Nery, 20(4), e20160099.

Lisanti, A. J., Cribben, J., Connock, E. M., \& Medoff-Cooper B. (2016). Developmental care rounds: an interdisciplinary approach to supportdevelopmentally appropriate care of infants born with complex congenital heart disease. Clinics Perinatology, 43(1), 147-56.

Magalhães, F. J., Lima, E. E. T., Rolim, K. M. C., Cardoso, M. V. L. M., Scherlock, M. S. M, \& Albuquerque, N. L. S. (2011). Respostas fisiológicas e comportamentais de recém-nascidos durante o manuseio em unidade de terapia intensiva neonatal. Revista Rene, 12(1):136-143.

Maki, M. T., Orsi, K. C. S. C., Tsunemi, M. H., Hallinan, M. P., Pinheiro, E. M., \& Avelar, A. F. M. (2017). O efeito da manipulação sobre o sono do recémnascido prematuro. Acta Paulista de Enfermagem, 30(5), 489-496

Marques, L. F., Ribeiro R. V., Rocha, C. R., Carreiro, M. A., \& Santiago, L. C. (2017). Cuidado ao prematuro extremo: mínimo manuseio e humanização. Revista de Pesquisa: Cuidado e Fundamental (online), 9(4), 927-931.

Marski, B. S. L., Facio, B. C., Ichisato, S. M. T., Barba, P. C. S. D., \& Wernet, M. (2018). Developmental Care: assistance of nurses from Neonatal Intensive Care Units. Revista Brasileira de Enfermagem, 71(6), 2758-2766.

Martins, L. A., Silveira, S. P. X., Avila, I. M. F. T., Moraes, J. A. S., Santos, D. S. S., Whitaker, C. O. M., \& Camargo, C. L. (2019). Implantação do protocolo de termorregulação para recém-nascido em procedimentos cirúrgicos. Revista Gaúcha de Enfermagem, 40, e20180218.

Mestrovic, J., Polic, B., Mestrovic, M., Kardum, G., Marusic, E., \& Sustic, A. (2008) Desfecho funcional de crianças tratadas em unidade de terapia intensiva. Jornal de Pediatria, 84(3): 232-236

Minayo, M. C. S. (2014). O desafio do conhecimento: pesquisa qualitativa em saúde. Hucitec Editora.

Ministério da Saúde. Conselho Nacional de Saúde (Brasil). Resolução nº 466, de 12 de dezembro de 2012. Decreto de Delegação de Competência. Diário Oficial da União 13 jun 2013, seção 1 .

Ministério da Saúde. Dados do Sistema Único de Saúde-DATASUS. Informações em Saúde. Nascidos vivos: Brasil. http://tabnet.datasus.gov.br/cgi/tabcgi.exe?sinasc/cnv/nvuf.def

Monteiro, L., Geremias, F., Martini, C., Vargas Makuch, D., \& Tonin, L. (2019). Benefícios do Toque Mínimo no Prematuro Extremo: Protocolo Baseado em Evidências. Revista Enfermagem Atual In Derme, 89(27).

Moody, C., Callahan, T. J., Aldrich, H., Gance-Cleveland, B., \& Sables-Baus, S. (2017). Early Initiation of Newborn Individualized Developmental Care and Assessment Program (NIDCAP) Reduces Length of Stay: A Quality Improvement Project. Journal of Pediatric Nursing, 32, 59-63.

Nascimento, L. C. N., Souza, T. V., Oliveira, I. C. S., Moraes, J. R. M. M., Aguiar, R. C. B., \& Silva, L. F. (2018). Theoretical saturation in qualitative research: an experience report in interview with schoolchildren. Revista Brasileira de Enfermagem, 71(1), 228-233

Nazario, A. P., Santos, V. C. B. J., Rossetto, E. G., Souza, S. N. D. H., Amorim, N. E. Z., \& Scochi, C. G. S. (2015). Avaliação dos ruídos em uma unidade neonatal de um hospital universitário. Semina: Ciências Biológicas e da Saúde, 36(1), 189-198.

Reis, L. S., Silva, E. F., Waterkemper, R., Lorenzini, E., \& Cecchetto, F. H. (2013). Percepção da equipe de enfermagem sobre humanização em unidade de tratamento intensivo neonatal e pediátrica. Revista Gaúcha de Enfermagem, 34(2), 118-124.

Ribeiro, J. F., Silva, L. L. C., Santos, I. L., Luz, V. E. S., \& Coêlho, D. M. M. (2016). O prematuro em unidade de terapia intensiva neonatal: a assistência do enfermeiro. Rev. enferm. UFPE on line, 10(10), 3833-3841.

Ribeiro, C. C., Pachelli, M. R. O., Amaral, N. C. O., \& Lamônica, D. A. C. (2017). Habilidades do desenvolvimento de crianças prematuras de baixo peso e muito baixo peso. CoDAS, 29(1), e20160058.

Rodarte, M. D. O., Fujinaga, C. I., Leite, A. M., Salla, C. M., Silva, C. G., \& Scochi, C. G. S. (2019). Exposição e reatividade do prematuro ao ruído em incubadora. CoDAS, 31(5), e20170233.

Rolim, K. M. C., Araújo, A. F. P. C., Campos, N. M. M., Lopes, S. S. M. B., Gurgel, E. P. B., \& Campos, A. C. S. (2010). Cuidado quanto à termorregulação do recém-nascido prematuro: o olhar da enfermeira, Rev. RENE, 11(2): 44-52.

Santana, L. S. R., Silva, L. S., Silva, R. R., Carvalho, J. E., Santana, W. S., Rossi-Barbosa, L. A. R., \& Ruas, E. F. G. (2015). Quantificação dos ruídos sonoros em uma unidade de terapia intensiva neonatal. Revista Mineira de Enfermagem, 19(2), 27-31. 
Research, Society and Development, v. 10, n. 4, e39010413993, 2021

(CC BY 4.0) | ISSN 2525-3409 | DOI: http://dx.doi.org/10.33448/rsd-v10i4.13993

Santos, A. M. G., Viera, C. S., Toso, B. R. G. O., Barreto, G. M. S., \& Souza, S. N. D. H. (2018). Clinical application of the Standard Operating Procedure of Positioning with Premature Infants. Revista Brasileira de Enfermagem, 71(3), 1205-1211.

Tong, A., Sainsbury, P., \& Craig J. (2007). Consolidated criteria for reporting qualitative research (COREQ): a 32-item checklist for interviews and focus groups. International Journal for Quality in Health Care, 19(6), 349 - 357. 